\title{
ESTUDO SOBRE A INFLUÊNCIA DO TEMPO DE EXPOSIÇÃO DO SUBSTRATO DE ALUMÍNIO AO TRATAMENTO POR PLASMA ELETROLÍTICO
}

\author{
Dannylo Soares Paiva* (Graduando em Ciência e Tecnologia na Universidade Federal Rural do Semi- \\ árido - UFERSA); \\ Hugo Fernandes Medeiros da Silva (Doutorando em Eng. Mecânica na Universidade Federal do Rio \\ Grande do Norte - UFRN);
}

Tarciana Dieb Toscano (Graduanda em Eng. Mecânica na Universidade Federal Rural do Semi-árido UFERSA);

Francisco Leonardo Gomes de Menezes (Técnico de Química na Universidade Federal Rural do Semiárido - UFERSA);

Gustavo Fábian Velardez; (Prof. de Química na Universidade Federal de Campina Grande - UFCG)

Clodomiro Alves Junior (Prof. Visitante Sênior na Universidade Federal Rural do Semi-árido -

UFERSA).

*Email: dannylopaiva@gmail.com

\section{RESUMO}

O tratamento de superfície dos materiais tem por finalidade conferir ao material novas características sem alterar suas propriedades internas e, como consequência disso, aumentar o campo de suas aplicações. Neste trabalho uma técnica relativamente nova foi utilizada para criar filmes finos de óxidos em alumínio, denominada Plasma Eletrolítico de Oxidação (PEO). Amostra de uma liga de alumínio foi tratada pelo processo de PEO, com diferentes tempos de exposição, que variaram de $600 \mathrm{~s}$ a 1200 s. Este trabalho objetivou melhorar o entendimento da técnica de PEO, além de observar e caracterizar a formação do filme que foi conferido a superfície do substrato metálico. No que tange a caracterização das amostras, foi realizada a Microscopia óptica, calculada a variação de peso das amostras, Microscopia Eletrônica de Varredura (MEV) e DRX com incidência rasante. Os resultados mostraram que o crescimento do filme é influenciado pelo tempo de exposição . A análise por microscopia óptica na amostra de $900 \mathrm{~s}$ mostra um importante crescimento do filme, apesar de não ter a maior quantidade de deposição. É também observado que o crescimento do filme é homogêneo.

Palavras chaves: tratamento de superfície; PEO; alumínio. 


\section{I NTRODUÇÃO}

O alumínio é o terceiro elemento metálico mais abundante na terra e devido sua baixa densidade, boa usinabilidade e excelente resistência à corrosão é um dos materiais mais utilizados na indústria (GONÇALVES, 2012). O alumínio tem sido um atrativo no segmento automobilístico por reduzir gastos com combustível (MCAULEY, 2003) devido seu peso leve em relação a de outros metais como o aço, além disso, com base na sua resistência física e à corrosão, permitelhe ser amplamente utilizado na indústria naval. Por outro lado, sua baixa dureza requer deste material um tratamento superficial diferenciado, afim de que seja concedido resistência suficiente tanto para a corrosão quanto para o desgaste.

O tratamento de superfície dos materiais tem por finalidade conferir ao material novas características sem alterar suas propriedades internas, e como consequência disso, aumentar o campo de suas aplicações. A utilização de revestimentos cerâmicos em metais tem se mostrado uma técnica bastante atrativa para a indústria por promover excelente resistência ao desgaste e a corrosão (ANTONIO, 2011).

Tem sido desenvolvida uma técnica relativamente nova para criar filmes finos de óxidos em metais leves, denominada Plasma Eletrolítico de Oxidação (PEO). O processo de PEO utiliza a mesma configuração da eletrólise convencional, mas é realizado a voltagens muito mais elevadas, geralmente na faixa de 400-700 V, e envolve um efeito de micro-descargas, que é apresentado em forma de faísca como resultado da ruptura dielétrica localizada no revestimento (MÉCUSON et al., 2005). Na tentativa de atender aos requisitos de sustentabilidade ambiental, o processo de PEO tem se mostrado um bom substituto dos métodos convencionais, já que a solução eletrolítica utilizada no processo não contém cromatos, metais pesados, solventes, ácidos ou outros componentes químicos tóxicos. Portanto, não exige tratamentos complexos antes de ser descartada e não oferece nenhum perigo durante seu manuseio.

Desta forma, o objetivo deste trabalho é melhorar o entendimento da técnica de Plasma Eletrolítico de Oxidação, para que possa ser controlado para produzir revestimentos com propriedades melhoradas, além de observar e caracterizar a formação do filme que foi conferido a superfície do substrato metálico.

\section{MATERI AIS E MÉTODOS}

A pesquisa foi conduzida no Laboratório LABPLASMA/UFERSA.

\subsection{Preparo das amostras}

As amostras foram produzidas a partir de uma placa de alumínio com as seguintes configurações dimensionais: $23 \mathrm{~mm} \times 23 \mathrm{~mm}$ x 0,7 mm conforme a Figura 1. Antes de começarmos o procedimento, o substrato passou por um processo de polimento com lixas na seguinte sequência granulométrica: 220, 400, 600, 1500 e 2000 micras. Em seguida, foi aplicado alumina em suspensão $0,05 \mu$ com pano de algodão em uma politriz lixadeira metalográfica até a superfície apresentar aparência espelhada. Afim de remover as impurezas e para garantir a ausência de resíduos, a amostra foi submetida a um processo de limpeza que consistia em lavá-la em uma cuba ultrassônica com solução de detergente por 660 segundos, em seguida foi enxaguada com água destilada e álcool $70 \%$. Após o término deste processo, a amostra foi seca utilizando um soprador térmico. Foram produzidas 5 amostras nesse arranjo. 


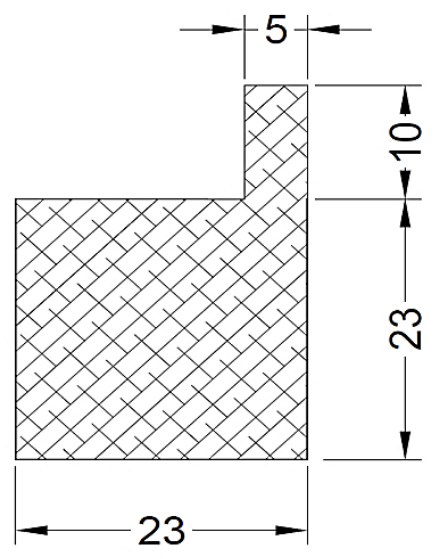

Figura 1

\subsection{Preparo da Solução}

Neste estudo, a solução eletrolítica utilizada foi o silicato de sódio $\left(\mathrm{Na}_{2} \mathrm{SiO}_{3}\right)$, com uma concentração de 1g/L. Com auxílio de uma balança analítica, pesou-se $1 \mathrm{~g}$ do eletrólito (silicato de sódio) e diluíu em 1 L de água destilada, com pH inicial de 11,8. Para cada tratamento foi utilizado aproximadamente $500 \mathrm{ml}$ de solução. A solução eletrolítica foi trocada a cada novo ensaio para que as condições de igualdade fossem garantidas.

\subsection{Aparato experimental}

O processo de tratamento foi realizado em um protótipo conforme ilustrado na Figura 2. Uma diferença de potencial foi aplicada entre os eletrodos, a tensão foi aumentada linearmente até atingir a condição de eletrolise a plasma, isto é, o aparecimento de centelhas em forma de microarcos. Estas movem-se rapidamente em torno da superfície da amostra e possui disposição uniforme além de apresentar coloração branca.

Cada amostra foi submetida a um tempo diferente de tratamento. Os tempos estabelecidos foram de: 600 s, 738 s, 900 s, 1020 s, 1200 s.

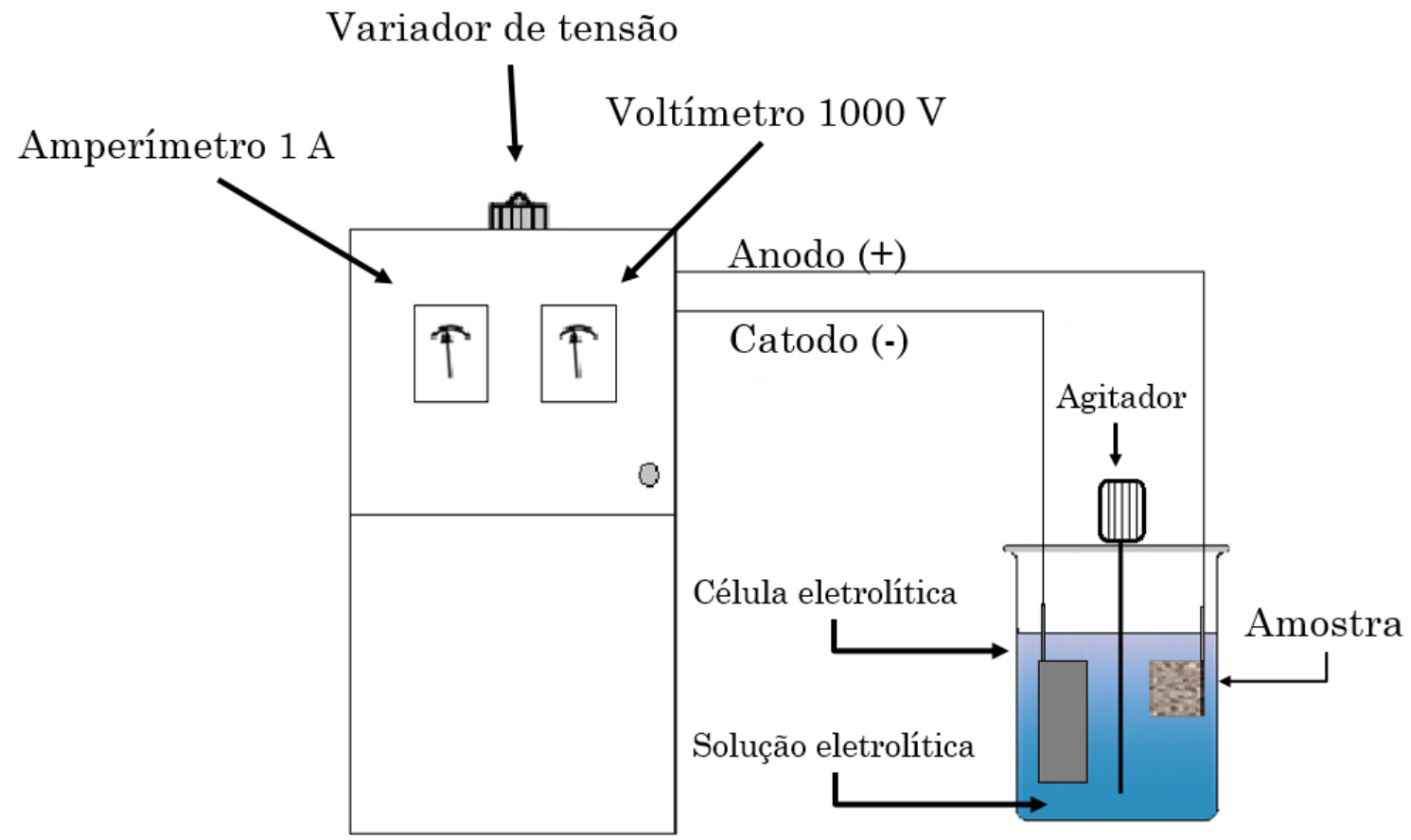

Figura 2 
No presente trabalho, verificou-se que para este equipamento a condição de geração de plasmas seria possível quando fosse estabelecida uma tensão no valor de $550 \mathrm{~V}$ e densidade de corrente de $0,08 \mathrm{~A} / \mathrm{cm}^{2}$. Os valores de densidade de corrente durante o processo PEO devem estar na faixa de 0,01 a $0,3 \mathrm{~A} / \mathrm{cm}^{2}$ (YEROKHIN, 1999), portanto o valor definido está dentro do intervalo.

As amostras foram pesadas antes e depois do tratamento para que se pudesse verificar variação de peso, em seguida foi analisada no MEV e por DRX com incidência rasante.

\section{RESULTADOS E DISCUSSÕES}

Verificada as condições necessárias e a eficácia do equipamento na geração do plasma em meio eletrolítico, iniciou-se o tratamento da amostra para os tempos previamente definidos. O substrato de interesse do estudo foi fixado no anodo, onde ocorre a descarga de plasma e a criação do filme de óxido na superfície da amostra.

\subsection{Microscopia óptica}

As imagens da Figura 3 realizadas nas amostras após o tratamento, foram obtidas através de um aumento de 1000x e são representações da região amostral do revestimento. Ao visualizar as micrografias, percebemos uma alteração superficial peculiar em cada amostra.
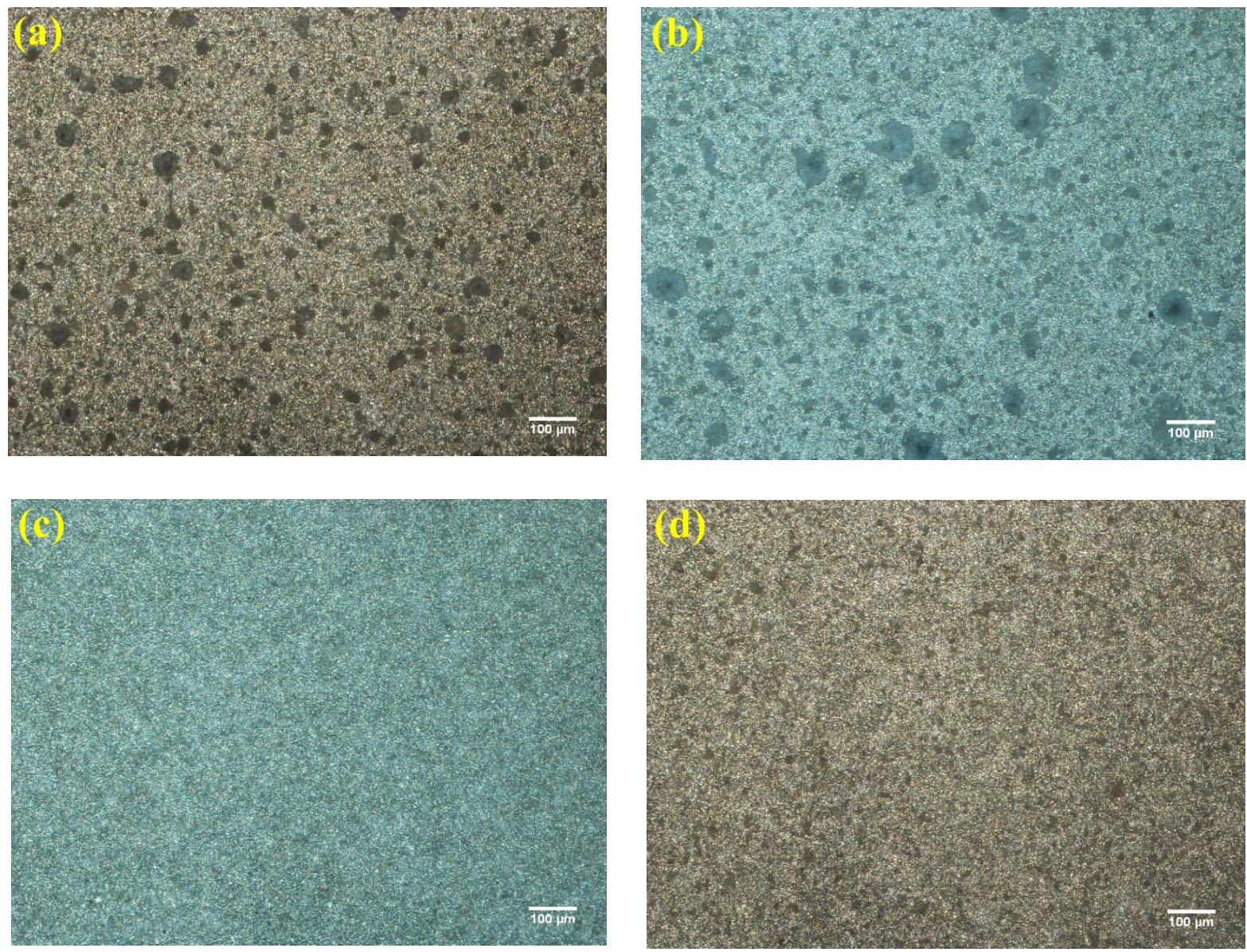


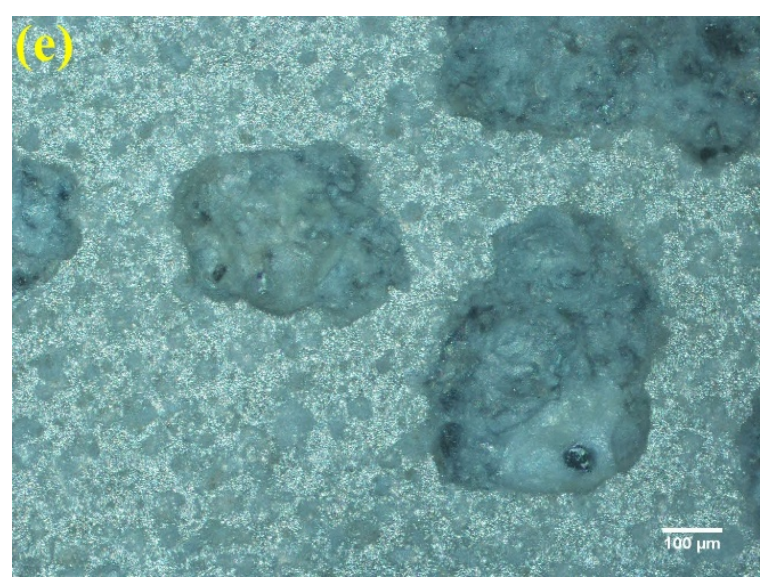

Figura 3 - Microscopia óptica, com ampliação de 1000x, após o tratamento PEO em diferentes tempos, sendo (a) $600 \mathrm{~s}$, (b) $738 \mathrm{~s}$, (c) $900 \mathrm{~s}$, (d) $1020 \mathrm{~s}$, (e) $1200 \mathrm{~s}$.

Na imagem (a) obtida com a amostra tratada por $600 \mathrm{~s}$, notam-se alguns pontos brancos e uma significativa quantidade de nódulos escuros, estes nódulos são partes residuais do substrato. Com o prolongamento do tempo de tratamento observamos que a quantidade de nódulos escuros foi diminuindo e a quantidade de pontos brancos foi aumentando, como pode ser verificado na imagem (b). Com o aumento do tempo ao tratamento PEO, os pontos brancos preenchem toda superfície. A imagem (c) correspondente a amostra tratada por $900 \mathrm{~s}$, mostra a superfície completamente preenchida pelos pontos brancos com ausência de nódulos. Na imagem (d) os pontos brancos ainda são maioria, entretanto, formações nodulares começam a aparecer. Com o passar do tempo de exposição das amostras ao tratamento, foi verificado arcos de alta potência, que segundo A. L. Yerokhin, perfuram a camada de revestimento até atingir e retirar parte do substrato provocando danos no revestimento, como pode ser verificado na imagem (e), estes arcos ainda são acompanhados de oscilações no valor da corrente.

\subsection{Variação de massa}

As amostras foram pesadas antes e depois do tratamento por PEO em uma balança de precisão.

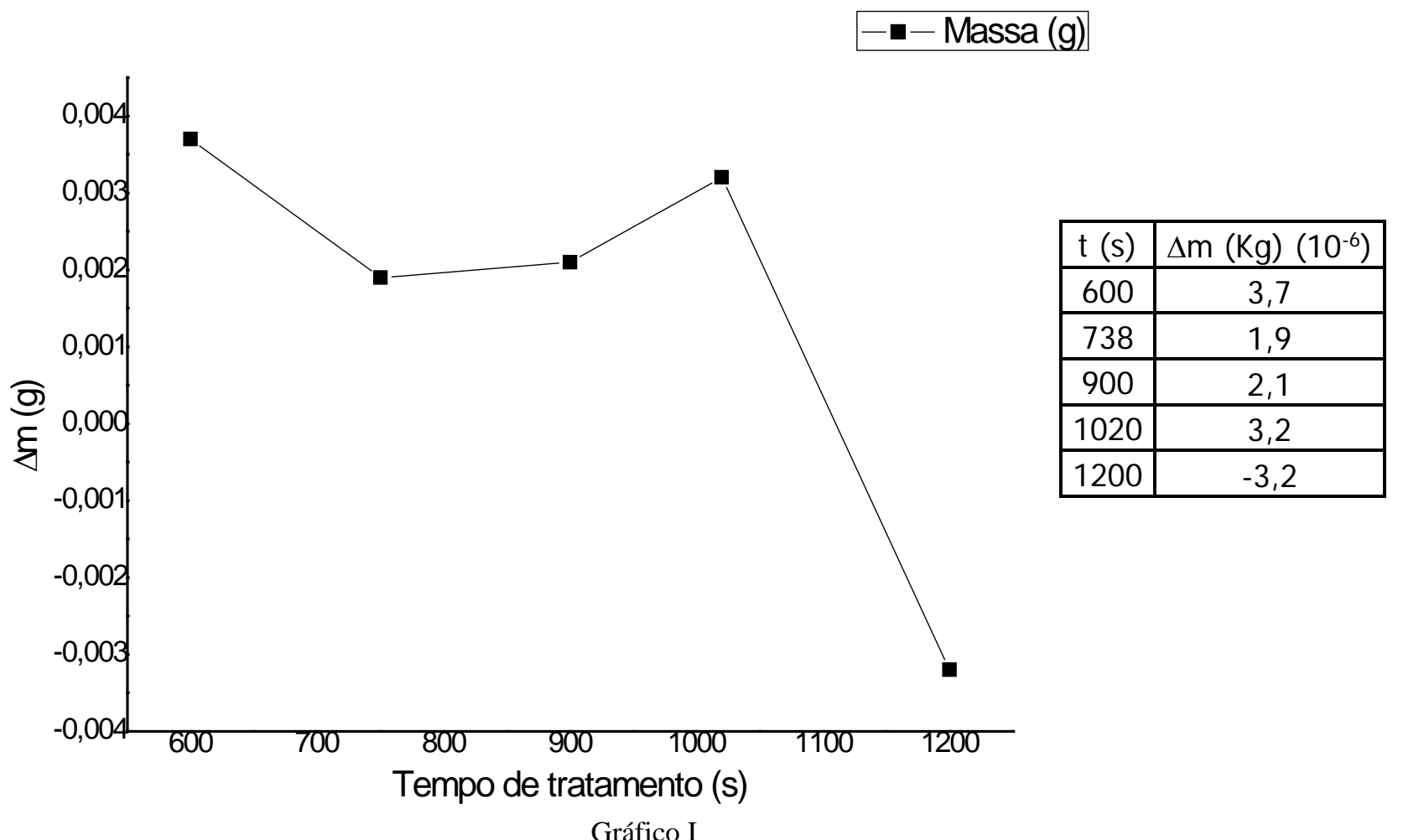


Pelo gráfico, pode-se observar que o tempo de $600 \mathrm{~s}$ foi o que obteve maior variação de massa, no entanto, esse aumento na massa não corresponde a deposição de material promovido pelo tratamento por PEO, e sim pela formação de um filme passivo correspondente ao potencial de oxidação do metal (YEROKHIN, 1999), pela Figura 3 (a) o filme de óxido não está completamente formado e apresenta regiões nodulares. De $600 \mathrm{~s}$ a $738 \mathrm{~s}$ temos uma queda abrupta no valor de variação de massa. Isso se deve ao fato de haver uma ruptura dielétrica no filme passivo de oxidação do metal e ter dado início a formação do filme de óxido. Em 900 s o filme de óxido teve seu crescimento contínuo e um ganho de massa significativo. Em 1020 s, observouse mais um pico na variação de massa, entretanto, essa deposição não se deu de forma uniforme como foi verificado na microscopia óptica, com a presença de nódulos escuros como pode ser constatado na Figura 3 (d). Em 1200 s, verificou-se uma perda considerável de massa, e isso é explicado pelos arcos terem adquirido alta potência térmica, penetrado o filme e extirpado parte do substrato, causando efeitos destrutivos.

\subsection{Microscopia Eletrônica de Varredura (MEV)}

Afim de avaliar o aspecto morfológico da deposição na superfície do substrato foi realizada a caracterização microestrutural através do MEV, conforme a Figura 4. Como observamos através da caracterização de microscopia óptica, a amostra de $900 \mathrm{~s}$ foi a que teve deposição mais uniforme e, por essa homogeneidade ser bastante atrativa quando falamos em filmes finos, esta amostra foi escolhida para fazer a MEV.
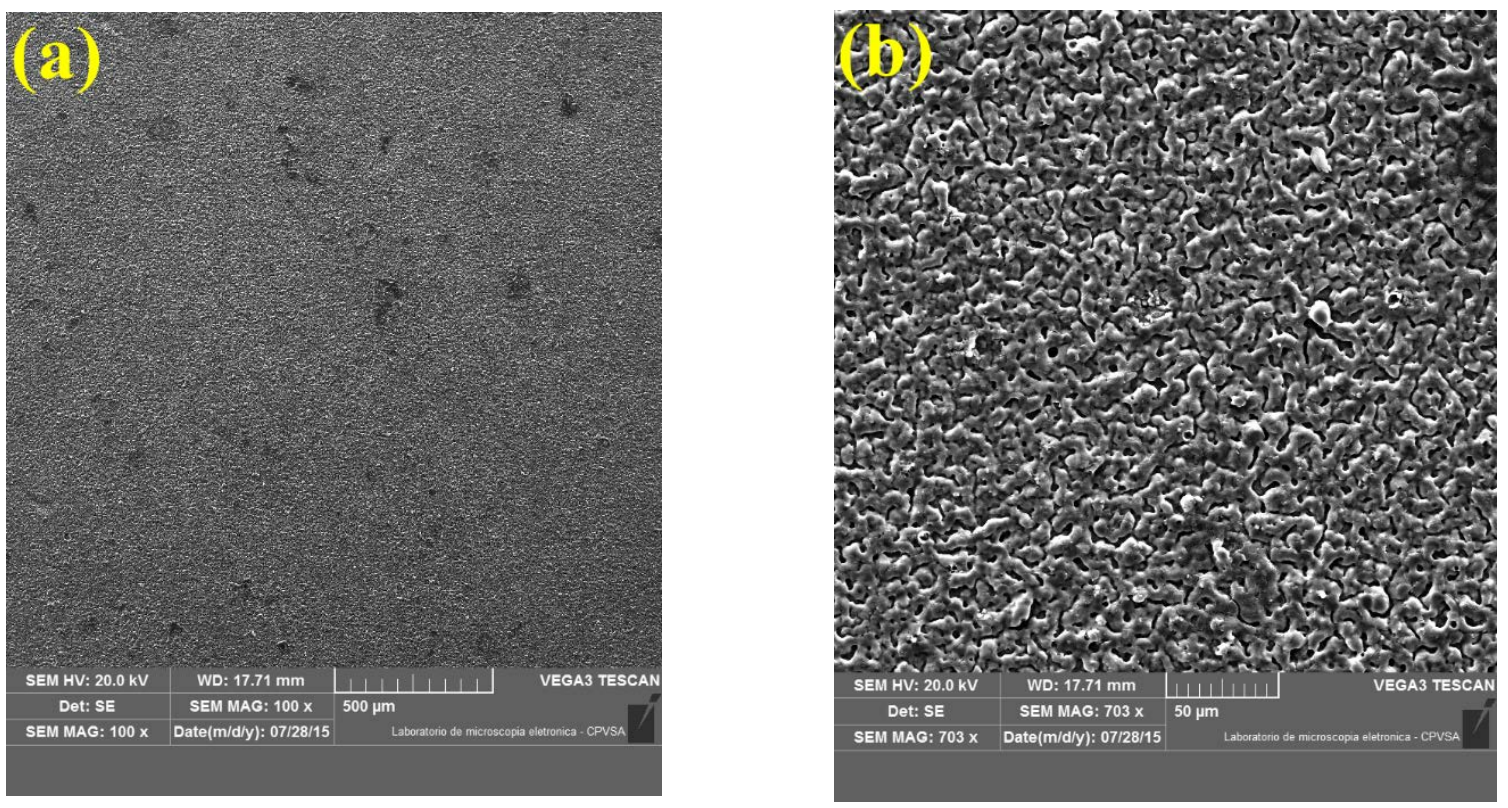


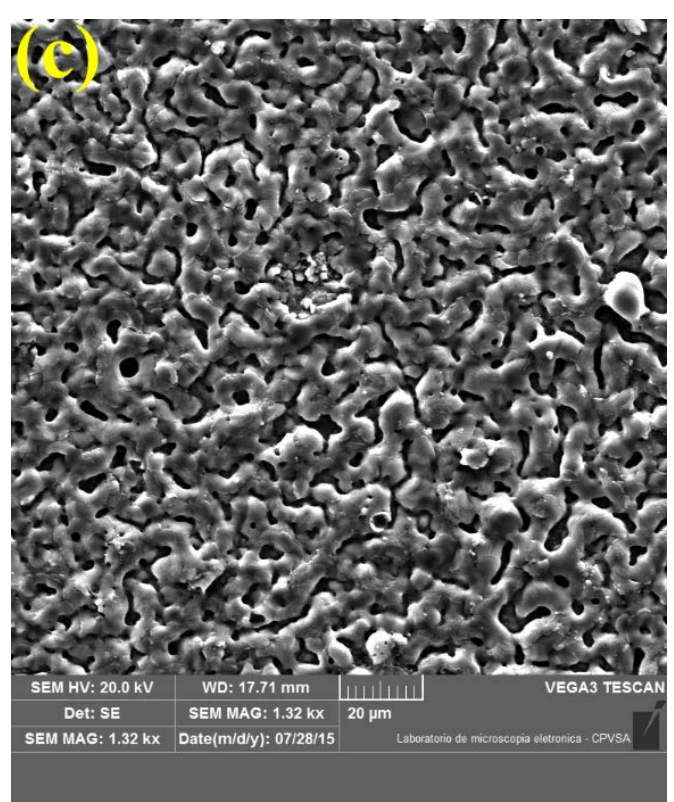

Figura 4 - Micrografia obtida por MEV, para tratamento por PEO pelo tempo de 900 s. sendo (a) aumento de 100x, (b) aumento de 703x e (c) $1.32 \mathrm{kx}$.

Ao examinar as micrografias observamos a alteração da morfologia superficial da amostra. Notoriamente, as imagens indicam uma formação homogênea de um fundo escuro e de pontos brancos.

\subsection{Difração de Raio X (DRX)}

Assim como no MEV, a amostra que realizamos o DRX foi a de $900 \mathrm{~s}$, por apresentar maior uniformidade. Pelo DRX de incidência rasante como mostra a Figura 5, os composto Óxido de Alumínio $\left(\mathrm{al}_{2} \mathrm{O}_{3}\right)$ e Alumínio (Al) foram observados.

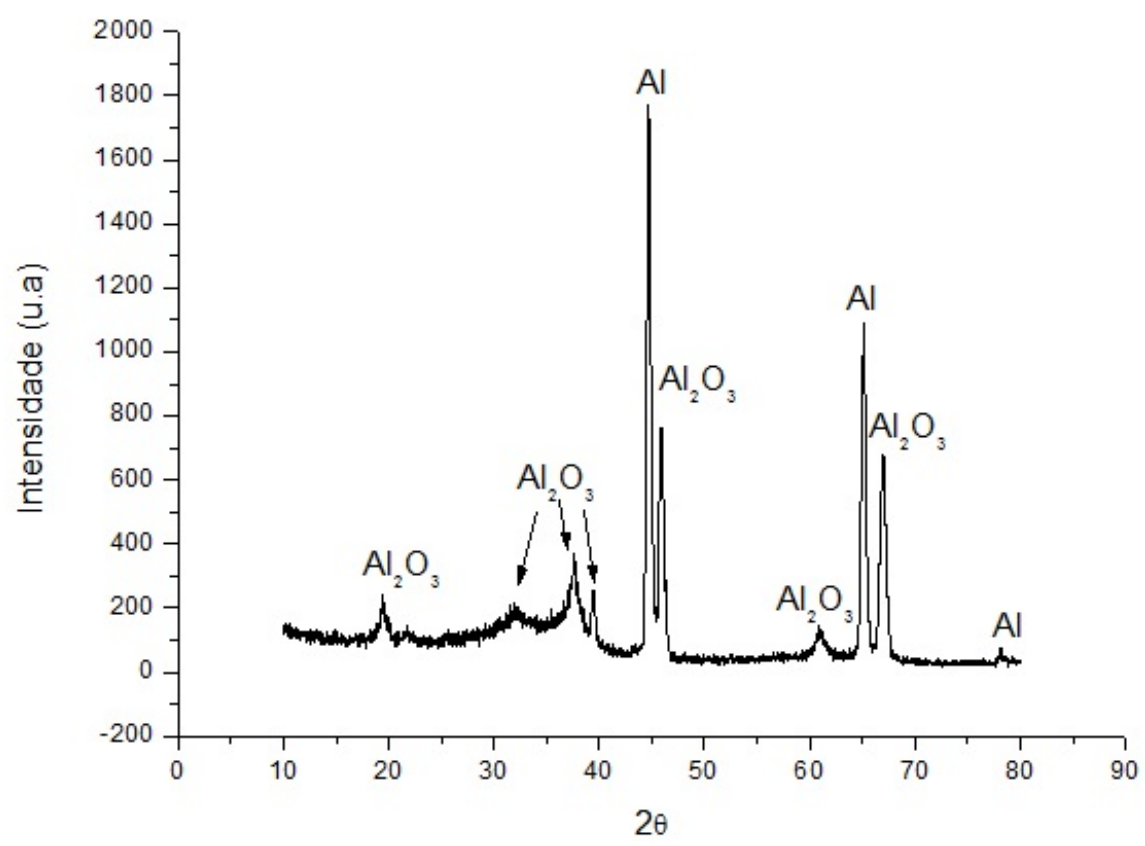

Figura 5 - DRX de incidência rasante da amostra de tratamento por PEO no tempo de 900 s. 


\subsection{Superfície do substrato}

Após o tratamento pode-se observar macroscopicamente que houve modificação na superfície do substrato, como podemos verificar na comparação entre as imagens Figura 6 (a) e (b). Sendo (a) amostra não tratada e (b) amostra após o tratamento de PEO por um período de 900 s. O revestimento obtido pelo tratamento por PEO em ligas de alumínio apresenta superfície porosa.
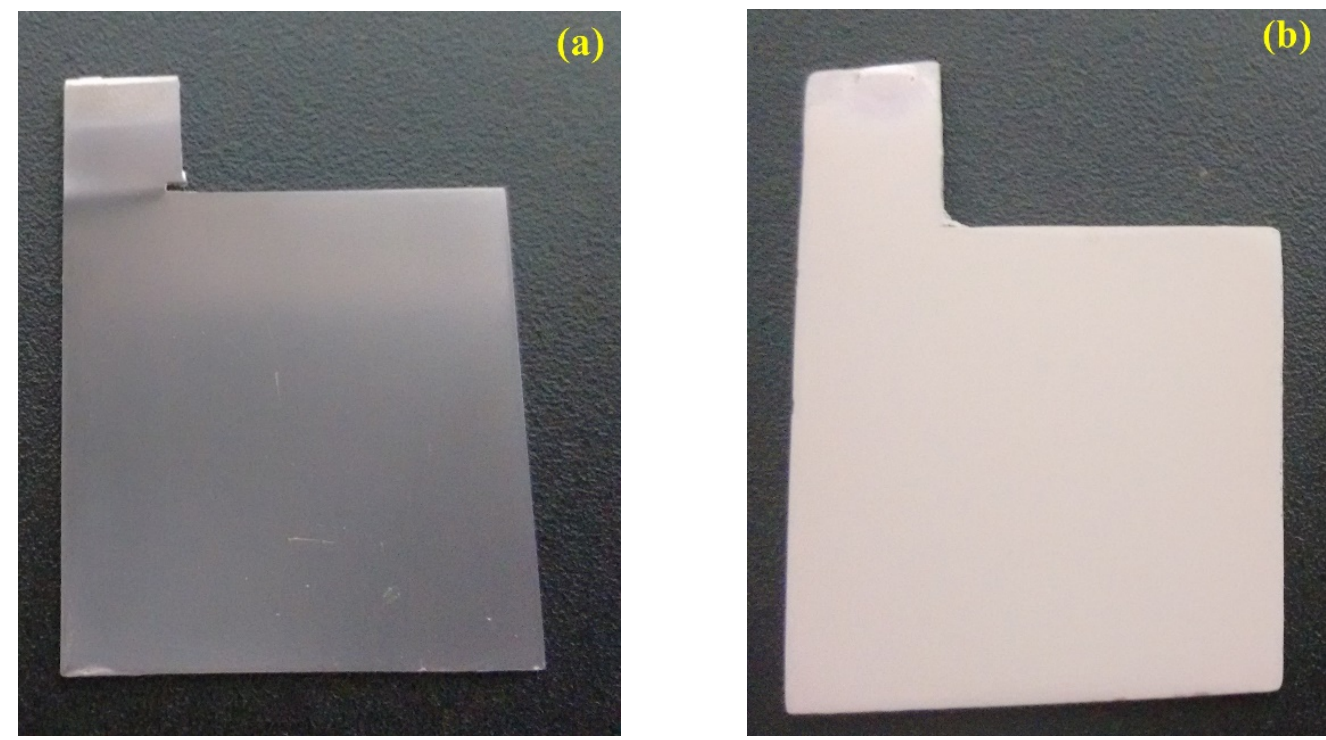

Figura 6 - (a) amostra não tratada (b) amostra tratada por PEO

Essa modificação superficial é atribuída ao mecanismo de formação de filmes por plasma eletrolítico que ocorre no revestimento de óxido de alumínio sobre a amostra. A eletrólise convencional proporciona o crescimento de um filme de óxido no substrato, o crescimento desse revestimento dá-se pela refusão da alumina e pela incorporação de elemento do meio eletrolítico. Pelo processo de oxidação do alumínio tem-se a formação da alumina que pode ser fundida através da ação dos micro-arcos e rapidamente refrigerada pela solução eletrolítica (XUE et al, 2000). Pelo processo eletrolítico, inicia-se o crescimento do revestimento cerâmico na superfície da amostra. Estes resultados estão diretamente relacionados com os parâmetros do processo aqui estudado. São eles, tensão x corrente em função do tempo, composição do eletrólito, assim como a geometria dos eletrodos. Com isso, podemos confrontar esses resultados com resultados encontrados na literatura que utilizam o mesmo processo para produção de filmes finos. O resultado do MEV apresenta uma estrutura nodular uniforme assim como em (DEHNAVI, 2014). No que tange o resultado de DRX, este apresentou um padrão de difração coerente com o que é apresentado em outro trabalho, onde em 900s obteve-se $\mathrm{Al}_{2} \mathrm{O}_{3}$ (OLIVERIA, 2010).

\section{CONCLUSÃO}

Através do processo de PEO, foi possível depositar um revestimento cerâmico sob o substrato. A técnica aplicada mostrou-se eficiente, pois houve um aumento significativo no peso das amostras, o que caracteriza que houve deposição, entretanto, na amostra de 1200 s houve perda significativa de material. A amostra de 900 s não foi a que teve maior ganho de deposição, todavia, houve um ganho significativo e pela microscopia óptica esta deu-se de forma bastante homogênea. $\mathrm{O}$ crescimento do revestimento na amostra ocorreu pelo processo de oxidação e por incorporação de elementos contidos na solução. Com a análise da microestrutura realizada no MEV, comprovou-se que o crescimento efetuou-se de forma uniforme e com o DRX de incidência rasante, observou-se os compostos esperados. Contudo, a amostra tratada por PEO pelo tempo de $900 \mathrm{~s}$ foi a que melhor apresentou características morfológicas. Vale ressaltar ainda que os filmes depositados mostraram uma interessante fixação sobre o substrato 
STUDY ON THE INFLUENCE OF TIME OS EXPOSURE TO THE PLASMA ELECTROLYTIC TREATMENT ON AN ALUMINUM SUBSTRATE

\begin{abstract}
Material surface treatment aims to provide the new characteristics of the material without changing its internal properties, and as a result, increases the reach of their applications. In this study, a relatively new technique called Plasma Electrolytic Oxidation (PEO) was used to ceramic thin films. Aluminum alloy samples was treated by PEO process for different exposition times, ranging from $600 \mathrm{~s}$ to $1200 \mathrm{~s}$. This study aimed to improve the investigation about the technique and surfaces properties. Regarding the characterization, optical microscopy, scanning electron microscopy and x-ray diffraction with grazing incidence was use. The results showed that the film growth is influenced by the exposure time. Analysis by optical microscopy at $900 \mathrm{~s}$ sample showed a significant growth of the film despite having the largest amount of deposition. It is also observed that the growth of the film is homogeneous.
\end{abstract}

Keywords: surface treatment; PEO; aluminum.

\title{
5. REFERENCIAS BI BLI OGRÁFI CAS
}

GONÇALVES, R. A. Investigação da Usinabilidade das Ligas de Alumínio da Série 6XXX. 2012. 107 f. Dissertação de Mestrado, Universidade Federal de Uberlândia, Uberlândia.

MCAULEY, J.W. Global sustainability and key needs in future automotive design, Environ. Sci. Technol. 37 (2003) 5414-6.

ANTONIO, C. A. DEPOSIÇÃO DE FILMES POR PLASMA ELETROLÍTICO EM LIGAS

DE ALUMÍNIO. Programa de Pos-graduacao em Ciencia e Tecnologia de Materiais (POSMAT) UNIVERSIDADE ESTADUAL PAULISTA (UNESP), 2011

MÉCUSON, F. CZERWIEC, T. BELMONTE, T. DUJARDIN, L. VIOLA, A. HENRION, G. Diagnostics of an electrolytic microarc process for aluminium alloy oxidation, Surf. Coatings Technol. 200 (2005) 804-808.

DEHNAVI, V. "Surface Modification of Aluminum Alloys by Plasma Electrolytic Oxidation" (2014). University of Western Ontario - Electronic Thesis and Dissertation Repository. Paper 2311.

YEROKHIN, A.L. et al. Review - Plasma Electrolysis for Surface Engineering, Surface and Coating Technology, v.122, p.73-93, 1999.

XUE W. et al. 2000; SUNDARARAJAN, G. et al. 2003 apud Wei-Chao Gu et al. Characterisation of ceramic coatings produced by plasma electrolytic oxidation of aluminum alloy, Materials Science and Engineering, v.A 447, p.158-162, 2007.

OLIVERIA, C. R. Alteração das Propriedades Superficiais do Alumínio via Eletrólise a Plasma, Programa de Pós-Graduação em Ciência e Tecnologia de Materiais, Universidade Estadual Paulista “Júlio de Mesquita Filho”, N. de páginas: 122. 2010. 